\title{
Indicators for interactive scheduling
}

\author{
Agnès Letouzey, Bernard Grabot and Laurent Geneste \\ LGP - ENIT, 47, avenue d'Azereix BP 1629, 65016 Tarbes cedex - France \\ Email: \{agnes, bernard, laurent\}@enit.fr
}

\begin{abstract}
In the present study, we suggest an approach of interactive scheduling based on the definition of various types of indicators. The purpose of these indicators is to help a workshop manager to assess the performance of a schedule but also to identify the best way for improving it.

The workshop manager uses the performance indicators to check whether the schedule satisfies the manufacturing objectives or not. If this satisfaction is considered as insufficient, diagnosis indicators can be used in order to identify usual causes of classical scheduling problems (e.g. bottlenecks). The workshop manager uses then action indicators to determine which improvement actions can lead to a significant improvement of the schedule quality. In that purpose, context indicators help to have first a global idea of the workshop state. The development of a software environment allowing to build these indicators using data contained in databases has been carried out during a European Project called "ASPIRE", and tests have been performed on the cases of companies belonging to the ASPIRE consortium.
\end{abstract}

\section{Keywords}

Interactive scheduling, indicators, dashboards.

\section{INTRODUCTION}

In order to meet customers expectations, companies must nowadays increase their competitiveness, by a better productivity and reactivity. Directly concerned by these needs, the scheduling function has to provide more and more reactivity regarding unexpected events, and has also to be highly adaptable regarding the definition of the schedule "quality". In that purpose, direct approaches, performing in one step a feasible and satisfying schedule are generally dedicated to specific applications. Iterative methods, consisting in improving progressively an existing schedule, have two main interests: they can include the user in the decisional loop, taking benefits of an expert knowledge which can be difficult to computerise, and they can remain generic while adapting to specific applications.

In next section a brief panorama of the evolution of the scheduling function and methods is presented. A global description of our approach of an interactive scheduling process is proposed. The types of indicators which are the base of this approach are described later. Paper concludes with the industrial application of our work in a European project called ASPIRE (CRAFT project BES2-5474). 


\section{FROM OPERATION RESEARCH TO INTERACTIVE SCHEDULING}

\section{Evolution of scheduling}

Scheduling has been for a long time a very active field of Operation Research, but the use of $\mathrm{OR}$ methods often requires modelling constraints poorly consistent with the huge set of technological constraints which have to be taken into account in order to ensure that the schedule is feasible (Farhoodi 1990, Smith 1992). In parallel, scheduling has progressively become a key function of the companies. A workshop manager, in charge of the schedule, tries to optimise both the service to the customer and the efficiency of the resource usage while trying to introduce the specificity of its workshop in a generic tool. An industrial scheduling software has now to address complex industrial needs, namely:

- to take into account particular resources, like ovens or painting tunnels,

- to respect more numerous and complex technological and temporal constraints,

- to satisfy several manufacturing objectives, sometimes conflicting,

a to be more reactive and more flexible.

Classical scheduling tools, often based on simple methods like list scheduling, cannot integrate all the specificities of resources and constraints in a given company, while optimising several objectives. Advanced scheduling tools, for instance based on Artificial Intelligence techniques, constraint propagation or fuzzy logic, are still too complex to be correctly mastered by workshop managers. Instead of increasing the complexity of the scheduling tools, we propose to introduce facilities allowing to progressively improve an existing schedule. In that case, an important economical challenge is to allow the use of present schedulers with only minor changes.

\section{Iterative approaches}

It has been frequently noticed (Farhoodi 1990, Grabot et al. 1999, Van Bael 1999) that a schedule is scarcely satisfactory at the first shot. It is generally useful to iteratively improve the schedule. The improvement of a schedule can be made automatically. It consists in starting from an existing solution, obtained by any means, and letting the scheduler improve it until obtaining a satisfying solution. This automatic improvement can be performed by Artificial Intelligence techniques, such as the scheduling systems presented in (Bensana et al. 1988, Ow and Smith 1988, Artiba and Arghezzaf 1997). The use of meta-heuristics, like Tabu search (Glover 1989 and 1990), genetic algorithms or simulated annealing (Metropolis et al. 1953), has also been considered to improve existing schedules, for instance in (Taillard 1994, Ponnambalam 1999, Van Bael 1999, Li et al. 2000, Chang \& 2001, Ponnambalam et al. 2001). Methods offering to automatically choose the best scheduling method or parameters according to the workshop characteristics, like in (Romanowicz et al. 1997, Pierreval and Mebarki 1997, Subramaniam et al. 2000), can be considered as automatic improvement techniques.

All these methods, aiming at automatically improve a schedule, are addressing a real industrial need. Nevertheless, they are scarcely integrated in commercial 
scheduling software. Indeed, their complexity and their running times are still repellent for companies. Moreover, if a workshop manager wants a schedule adapted to his requirements, he also wants to control its adaptation. A totally automatic improvement process will cut him off from the schedule and can lead him to reject it. Furthermore, it has been often stated, like in (Baek et al. 1999), that the workshop manager is usually more efficient for improving a schedule than automatic methods. A better way for improving a schedule appears to include the workshop manager in the improvement loop. Cooperative scheduling is defined as the common achievement of the schedule by the software and the workshop manager. This cooperation allows the user to be really involved in the scheduling process and consequently to adapt the schedule to his needs and to appropriate the result. Schedule improvement methods based on cooperative scheduling have for instance been suggested in (Lopez et al. 1998) or (Grabot et al. 1999). However, this implication requires a very good understanding of the scheduling method used by the scheduling software. Interactive scheduling is defined in (Farhoodi 1990) as an alternation of decisions of the operator and the software through the following steps, as shown in Figure 1:

- input data (user),

- scheduling (software),

- results visualisation (software),

- results evaluation (user).

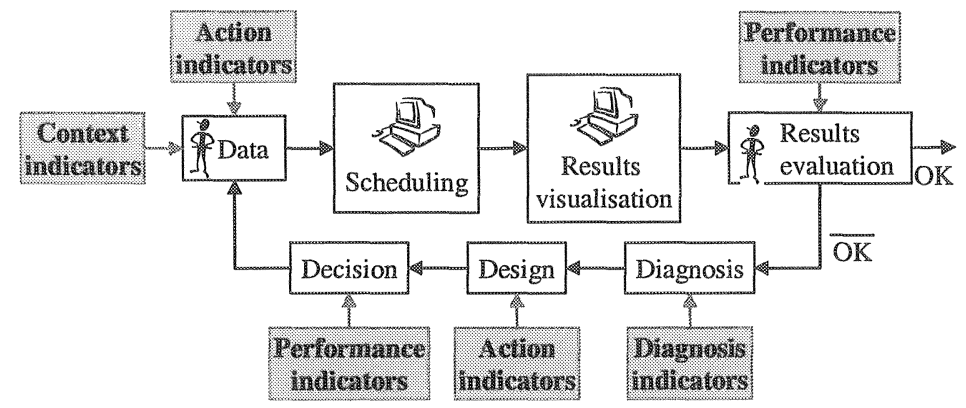

Figure 1 - Indicators in interactive scheduling

This approach is a possible answer to the industrial requirements. We suggest here the definition of a set of indicator classes aiming at improving the integration of the human decision maker in the improvement loop.

\section{INDICATORS FOR INTERACTIVE SCHEDULING}

\section{Indicator definition}

Performance indicators are defined in (AFGI 1992) as "quantified data that measure the efficacy and/or efficiency of the whole or part of a process or a system, as compared to a norm, a plan or an objective, determined and accepted in the frame 
of a company strategy." This definition emphasises different characteristics of the performance indicators, formalised for instance in (Berrah et al. 2000):

- an indicator has to be quantified, to measure something,

- an indicator is related to an objective, which satisfaction is assessed,

- an indicator is linked to the actions, which can increase the objective satisfaction.

Therefore, an indicator is composed of three fundamental facets and can be considered as a triplet \{objective, measure, action\} (Bitton 1990). A performance indicator can also be considered as qualitative. In that case, it can be assessed on an ordinal scale (simply by ranking the different possibilities) or evaluated with the use of fuzzy logic (Zadeh 1965).

\section{Interactive scheduling and Indicators}

Improving the performance of a system requires first to have a measure on the distance of its actual behaviour from the expected one: this is for instance the base of automatic control and requires the definition of performance indicators. As a matter of fact, performance indicators only provide the first step of the improvement loop: many actions are possible in present industrial schedulers, and making this loop more efficient requires to support the user in the choice of the best possible action in a given context. Our objective is to provide the user with an assistance in order to evaluate the schedule performance, but also to assess the different actions that could improve the schedule, as shown in Figure 1.

\section{Context indicators}

Context indicators are used to interpret the global state of the workshop when a schedule is about to be performed. They are not related to any objective nor to any action that could increase the objective satisfaction. Context indicators are composed of a simple measure (qualitative or quantitative). They can concern inventories levels for components, tools or raw materials, be related to the load (number of planned manufacturing orders, number of operations planned or being processed, number of tasks manufactured outside...) or to the capacity (number of workers present, missing, on vacations or sick, number of machines available, used or out of order...).

\section{Diagnosis indicators}

Diagnosis indicators aim at helping the workshop manager to find the causes of a problem. These indicators should be quite simple and are not supposed to be complex diagnosis tools like Ishikawa diagrams or AMDEC analysis. This kind of indicator is used to identify classical causes of usual scheduling problems, e.g. global overloading (load/capacity balance of the overall workshop), local overloading, i.e. bottlenecks (load/capacity balance on each machine), lack of capacity (unavailability of required resources) etc. 


\section{Action indicators}

Action indicators guide the user of the scheduling software towards a feasible and efficient solution. They allow to differentiate the different types of actions:

a impossible actions, which can not be performed in the present context,

w feasible actions, which can be launched,

m interesting actions, which are feasible and should lead to increase the system performances.

Action indicators give information to the scheduling software user about which degrees of freedom can be used and how relevant they are in the scheduling context. Thus, an action indicator is not a simple information, but is composed of a measure (the information it gives about the workshop and/or the scheduling context) and an action (the degree of freedom which use is evaluated). Different types of action indicators can be considered:

- action indicators about the existence of the degree of freedom which use is considered: other dispatching rules, possibility to manually move tasks or orders, availability of alternative resources, operations or routings...

- action indicators about the distribution of a characteristic of the workshop. In order to know if an action can lead to an interesting result, it is often important to know whether the distribution of the characteristic on which the action is based is uniformly distributed over the workshop or not. For example, if the workshop manager wants to use a dispatching rule based on the priority of the order, it is interesting to know whether the priority of the orders is uniform or if most of the orders have already a high priority.

a action indicators about degrees of freedom robustness. When two alternative actions are considered, which should both lead to an interesting increase of the schedule performances, it can be useful to provide the workshop manager with information about the robustness of both solutions, i.e. which solution should remain efficient through time.

\section{Performance indicators}

Performance indicators are assessing the schedule performed by the scheduling software, guided by the workshop manager, regarding the company objectives. The objectives assigned to the scheduling function come from the enterprise global objectives by a hierarchical decomposition (Farhoodi 1990, Grabot 1998). Performance indicators evaluate the difference between the measured state of the system and the objective assigned. Performance indicators are composed of:

- a measure (real state of the schedule),

- an objective (desired state of the schedule),

- if possible, action indicators (usual points of action leading to an improvement of this performance).

These triplets have to be progressively built by the workshop manager, based on his knowledge about the workshop and the scheduling software. Performance indicators, in a scheduling context, can be classified into four classes:

- temporal indicators, 
* resources indicators,

- financial indicators,

others.

\section{Interactive scheduling}

Figure 1 shows how these different types of indicators intervene in the interactive scheduling process. For a first schedule (asked periodically), the workshop manager uses context indicators to describe the global state of the workshop and action indicators to start the scheduling process with a good configuration of the scheduler parameters. When the schedule is performed by the software, the user evaluates its performances with performance indicators. If these performances are acceptable, the schedule is applied into the workshop. On the contrary, the workshop manager tries to identify the causes of these bad performances with diagnosis indicators. The next step consists in finding the different possible solutions (set of actions which change some scheduling parameters and some workshop data in order to improve the schedule performances), with the assistance of action indicators. These different solutions are assessed by performance indicators to help the workshop manager to choose the "best" solution.

All these different types of indicators are usually visualised on dashboards (a dashboard being a synthetic visualisation of indicators needed to evaluate a situation). Dashboards can be composed by performance (one performance indicator and its action indicators), or by problem (one performance indicator that detects the problem, diagnosis indicators which find the causes of the problem and action indicators which help to solve the problem). In order to improve a schedule, the problem is not so much to choose a dashboard than to define the sequence of different dashboards which will be used within the different steps leading to this improvement.

\section{APPLICATION: THE ASPIRE PROJECT}

This approach of interactive scheduling process based on the use of indicators has been implemented in a tool developed within the ASPIRE project.

\section{ASPIRE Project}

ASPIRE (Anticipated SME Production Simulation for Negotiation Support within a Customer/Supplier Context) is a European CRAFT project, which aimed at developing a set of software tools allowing a sub-contractor to forecast its lead time during the negotiation phase with a customer, then to schedule the order when it is confirmed. The ASPIRE consortium was the following:

- five SMEs (Small and Medium Enterprises) from various production areas (mechanical parts for aeronautics (MAP, France), other mechanical parts (De Nadaï, France), cutting tools (Ebay, Spain), technical textiles (Textinap, France), off-set printing (Krammer, Austria), 
" two research laboratories (BIBA, Germany; LGP, France),

a two Information Technology vendors (Robotiker, Spain; IXI, France).

Within the consortium, the role of the SMEs was to express their requirements, then test and validate the developed system while the research laboratories and IT vendors had to specify, design and develop the software package. The ASPIRE tool is composed of four modules:

a a repository module, centralizing all the data (from the workshop and from the other modules),

ఐ a macro-scheduling module, performing a rough schedule,

- a simulation module, performing a precise schedule according to different scenarios,

- an assessment module.

\section{Assessment module of the ASPIRE tool}

The assessment module of the ASPIRE tool is dedicated to the evaluation of the macro-schedule or the simulation results. It allows to interactively build dashboards containing the different types of indicators. This module is composed of six submodules as shown in Figure 2.

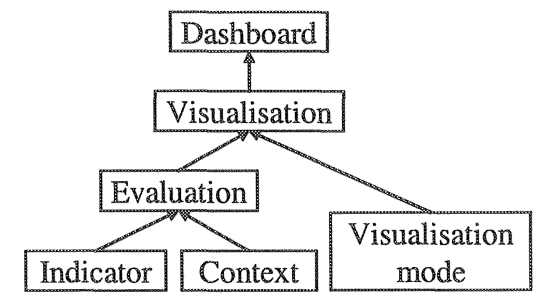

Figure 2 - Assessment module architecture

The "indicator" sub-module allows to define way to calculate the indicators. Three types of indicators are proposed:

- elementary indicators, obtained directly from the database, e.g. lateness of an order,

* aggregated indicators, which aggregate in one synthetic indicator the application of one indicator on several objects, e.g. average lateness of all the orders,

" combined indicators, aggregating several different indicators in one, e.g. utilisation ratio of a machine, resulting of the ratio between working and open hours of a machine, which have both to be defined as elementary objectives since they have to be separately calculated from data present in the database.

The "context" sub-module allows to choose the objects on which an indicator will be applied (it defines so a filter): e.g. for the lateness: "all the orders", "orders of priority $>10$ ", "orders for Customer 1". The "evaluation" sub-module allows to associate an indicator and a context, which means to apply the indicator equation on the objects selected by the context. The "visualisation mode" allows to choose the different parameters of the visualisation (type, colours, fonts...). In the 
"visualisation" sub-module, it is possible to associate a visualisation mode with an evaluation, i.e. to calculate and visualise the indicator. Finally, the "dashboard" submodule allows to build dashboards, that means choosing the set of visualisations, with their position and size, that the dashboard will contain.

Three levels have been defined for using the Assessment tool:

- level 1: the user can only use pre-defined indicators, chosen in lists, to define his own dashboards,

- level 2: the user can define his own indicators and their aggregation using a graphical user interface (GUI) (by selecting attributes of the database and operators in menus),

w level 3: when very specific operators have to be defined that can not be described using the pre-defined functions of the GUI, the indicators and their aggregation can directly be defined using SQL queries.

At the second level, the user can define his own indicators and contexts, by defining their equations. These equations are entered through a GUI as trees, a tree being composed of nodes (arithmetic operators) and leaves (database attributes and/or other indicators). For example, the filter "orders whose priority is superior to $10^{\prime \prime}$ is defined by the equation (order_priority $>10$ ), entered as the tree shown in Figure 3.

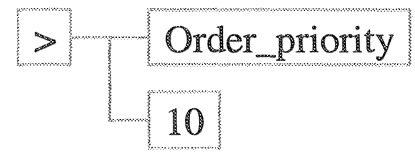

Figure 3 - Tree defining the equation (order . priority > 10)

Figure 4 shows the GUI allowing to build a tree defining an indicator. The equation here corresponds to the lateness and is defined as (planned_end_date delivery_date). 


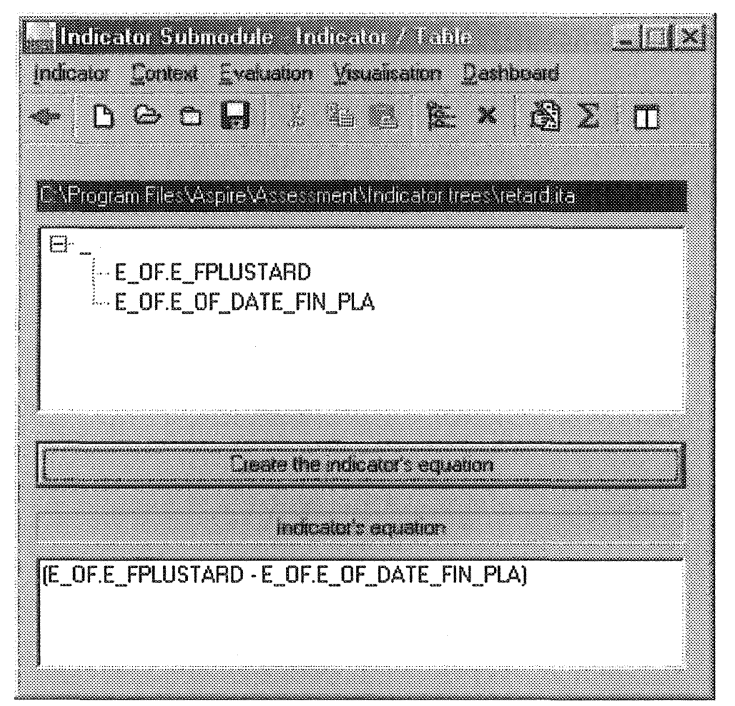

Figure 4 - Definition of an indicator in ASPIRE assessment tool

The assessment module provides a generic way to structure and visualise the information present in a database: it is therefore possible to use this module with any scheduling software.

\section{Example}

In Figure 5, an example of dashboard achieved on a schedule performed by a first range industrial scheduler is shown. This dashboard is build according to the performance of the schedule regarding the lateness of the orders. At the centre of the dashboard, a performance indicator shows the average lateness of all the manufacturing orders. At the top of the dashboard, two diagnosis indicators help to find the cause of the lateness shown by the performance indicator:

- resources utilisation rate:

sum of the duration of the tasks performed on the machine

" orders efficiency:

Cycle time of the order

sum of the duration of the tasks composing the order

$$
\text { Cycle time of the order }
$$

It appears that the lateness comes from a bottleneck ( $\mathrm{TCN}$ resource). These indicators are considered here as diagnosis indicators, as they help in finding the cause of the observed lateness. In other dashboards, dedicated for example to the objective "maximise the resources utilisation", they can be considered as performance indicators.

In order to decrease the lateness, the workshop manager can consider the change of the dispatching rule of that resource. Two rules can be chosen: one about the margin of the orders and one about tasks duration (SPT or LPT for instance). The 
first action indicator, at the left bottom of the dashboard, measures the distribution of the orders margins, while the second action indicator, at the right bottom, measures the distribution of the tasks duration. The first one shows that the first considered rule will not lead to an interesting change as all the orders have about the same margin, while the second one shows that changing to LPT or SPT can be more interesting.

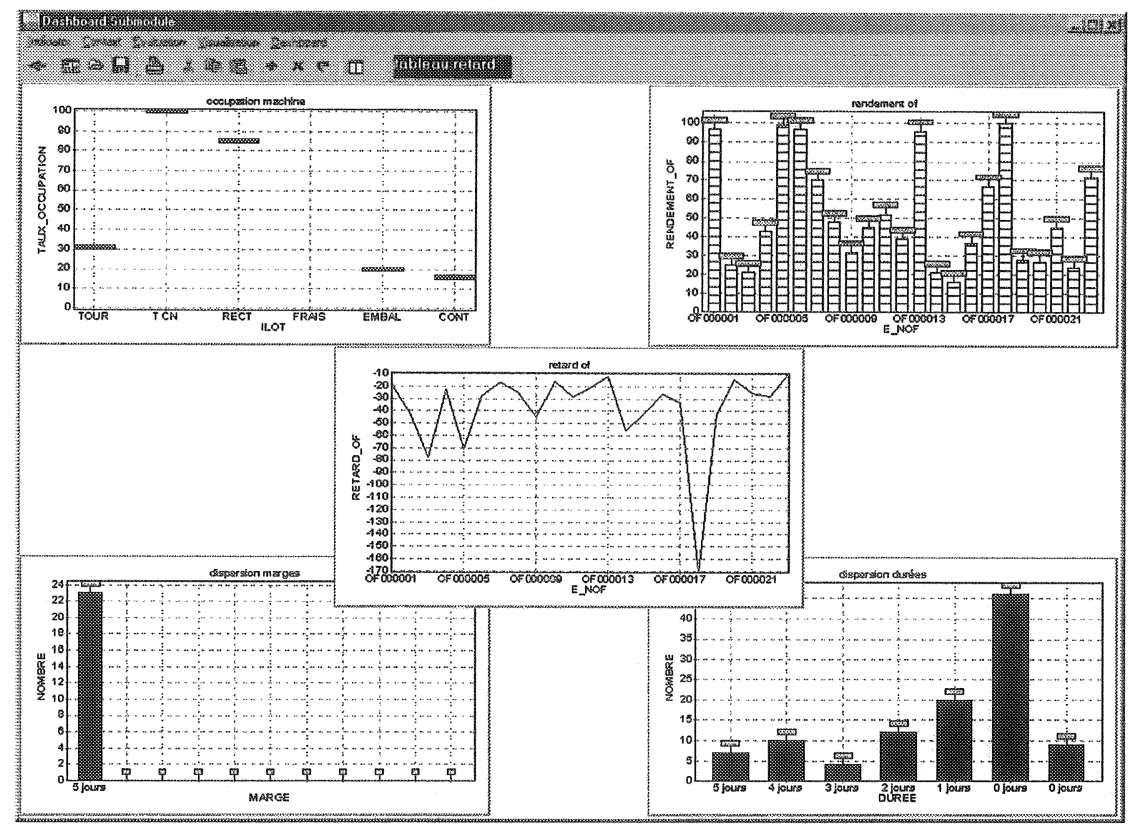

Figure 5-Example of dashboard

\section{CONCLUSIONS}

In this paper an approach of interactive scheduling based on the use of indicators is presented. This approach allows a workshop manager to improve a schedule and supports him through this improvement by assessing the different possible actions. Four types of indicators have been defined: context indicators, diagnosis indicators, action indicators and performance indicators. A tool allowing to build dashboards has been developed during a European project called ASPIRE. These dashboards allow to visualise the different types of indicators, and then to perform the evaluations needed in interactive scheduling.

The dashboards built using the ASPIRE tool are still experimental and the next step of our study is then to test our approach in a real industrial environment, by creating dashboards, with the help of a workshop manager, dedicated to his workshop.

This interactive approach to scheduling, based on the definition of indicators, is interesting as it can adapt to any company specificities and offers the possibility to perform multi-objective schedules. It is also interesting to treat particular and 
complex cases, such as manpower management or maintenance planning. The definition of complex indicators, and possibly of treatment, can help in solving these peculiar problems.

\section{REFERENCES}

[1] Artiba and E.H. Aghezzaf, 1997, "An architecture of a multi-model system for planning and scheduling", International Journal of Computer Integrated Manufacturing, vol. 10, n'5, pp. 380 - 393.

[2] Association Française de Gestion Industrielle, 1992, Evaluer pour évoluer, les indicateurs de performance au service du pilotage industriel, collective book.

[3] D.C. Baek, S.Y. Oh and W.C. Yoon, 1999, "A vizualized human-computer interactive approach to job shop scheduling", International Journal of Computer Integrated Manufacturing, vol. $12, n^{\circ} 1$, pp. $75-83$.

[4] E. Bensana, G. Bel and D. Dubois, 1988, "OPAL: a multi-knowledge based system for industrial job-shop scheduling”, International Journal of Production Research, vol. 26, $n^{\circ} 3$, pp. $795-815$.

[5] L. Berrah, G. Mauris, L. Foulloy and A. Haurat, 2000, "Global vision and performance indicators for an industrial improvement approach", Computer in Industry, $\mathrm{n}^{\circ} 43$, pp. 211-225.

[6] M. Bitton, 1990, "ECOGRAI : méthode de conception et d'implantation des systèmes de mesure des performances pour organisations industrielles", Ph.D. Thesis, Bordeaux I University, France.

[7] P.T. Chang and Y.T. Lo, 2001, "Modelling of job shop scheduling with multiple quantitative and qualitative objectives and a GA/TS mixture approach", International Journal of Computer Integrated Manufacturing, vol. 14, n4, pp. 367 - 384.

[8] P. Esquirol and P. Lopez, 1999, L'ordonnancement, Economica.

[9] F. Farhoodi, 1990, "A knowledged-based approach to dynamic job-shop scheduling", International Journal of Computer Integrated Manufacturing, vol. 3, n², pp. 84 - 95.

[10] F. Glover, 1989, "Tabu search, part 1", Orsa Journal on Computing, vol. 1, n³, pp. 190 $-206$.

[11] F. Glover, 1990, "Tabu search, part 2", Orsa Journal on Computing, vol. 2, n¹, pp. 4 32.

[12] Grabot, 1998, "Objective satisfaction assessment using neural nets for balancing multiple objectives", International Journal of Production Research, vol. 36, n9, pp. $2377-2395$.

[13] Grabot, C. Bérard and P. Nguyen, 1999, "An implementation of man-software cooperative scheduling: the IO software", Production Planning and Control, vol. $10, n^{\circ} 3$, pp. $238-250$.

[14] Y. Li, K.F. Man, K.S. Tang, S. Kwong and W.H. Ip, 2000, "Genetic algorithm to production planning and scheduling problems for manufacturing systems", Production Planning and Control, vol. 11, n`5, pp. $443-458$.

[15] P. Lopez, P. Esquirol, L. Haudot and M. Sicard, 1998, "Cooperative system design in scheduling", International Journal of Production Research, vol. 36, n¹, pp. $211-230$.

[16] N.A. Metropolis, a. Rosenbluth, A. Teller and E. Teller, 1953, "Equation of state calculations by fast computing machines", Journal of Chemestry and Physics, vol. 6, $\mathrm{n}^{\circ} 21$.

[17] P.S. Ow and S.F. Smith, 1988, "Viewing scheduling as an opportunistic problem solving process”, Annals of Operations Research, vol. 12, pp. 85 - 108. 
[18] H. Pierreval and N. Mebarki, 1997, "Dynamic selection of dispatching rules for manufacturing systems scheduling" International Journal of Production Research, vol. $35, \mathrm{n}^{\circ} 6, \mathrm{pp} .1575-1591$.

[19] S.G. Ponnambalam, N. Jawahar and P. Aravindan, 1999, "A simulated annealing algorithm for job shop scheduling", Production Planning and Control, vol. 10, $n^{\circ} 8$, pp. $767-777$.

[20] S.G. Ponnambalam, P. Aravindan and S. Chandrasekaran, 2001, "Constructive and improvement flow shop heuristics: an extensive evaluation", Production Planning and Control, vol. $12, n^{\circ} 4$, pp. $335-344$.

[21] R. Romanowicz, J. Jacot, A. Hertz and E. Verdebout, 1997, "An expert system prototype for the selection of the scheduling method", ETFA'97, $6^{\text {th }}$ International Conference on Emerging Technologies and Factory Automation, Los Angeles, USA, September $9^{\text {th }}-12^{\text {th }}$.

[22] S.F. Smith, 1992, "Knowledge-Base Production Management: Approaches, Results and Prospectives", Production Planning and Control, vol. 4, n⿳3, pp 350 - 380.

[23] H.A. Simon, 1977, The new science of management decision, Prentice-Hall.

[24] V. Subramaniam, G.K. Lee, G.S. Hong, Y.S. Wong and T. Ramesh, 2000, "Dynamic selection of dispatching rules for job shop scheduling", Production Planning and Control, vol. $11, \mathrm{n}^{\circ} 1$, pp. $73-81$.

[25] Taillard, 1994, "Parallel tabu search technique for the job shop scheduling problem", Orsa Journal on Computing, vol. 6.

[26] P. Van Bael, 1999, "A study of rescheduling strategy and abstraction levels for a chemical process scheduling problem", Production Planning and Control, vol. 10, $\mathrm{n}^{\circ} 4$, pp. 388 - 398.

[27] L.A. Zadeh, 1965, "Fuzzy Sets", Information and Control, vol.8, pp. 338 - 353. 